Pathologe 2010 - [Suppl 2] 31:258-262

DOI 10.1007/s00292-010-1336-8

Online publiziert: 15. August 2010

(c) Springer-Verlag 2010

\section{S. Diederichs}

Helmholtz-Hochschul-Gruppe „Molekulare RNA Biologie \& Krebs“, Deutsches

Krebsforschungszentrum (DKFZ) \& Pathologisches Institut Universität Heidelberg

\title{
Nichtcodierende RNA in malignen Tumoren
}

\section{Eine neue Welt von Tumor-Biomarkern und Zielstrukturen in Krebszellen}

Die Gesamtheit der menschlichen Erbinformation enthält etwa 20.000 proteincodierende Gene. Diese Gene entsprechen dem klassischen Dogma der Molekularbiologie: Sie werden in Boten- („messenger"-) RNA (mRNA) transkribiert, diese wird in Proteine translatiert, welche dann eine Funktion der Zelle ausführen. Neuste Untersuchungsmethoden zeigen aber, dass das Genom nicht nur die Proteinbaupläne enthält, sondern dass viel größere Teile des Genoms in nichtproteincodierende RNA transkribiert werden. Diese nichtcodierenden RNA-Moleküle (ncR-
NA) stellen daher eine wichtige Quelle für neue Biomarker dar. Eine bekannte Klasse von ncRNAs sind die kurzen microRNAs, die wichtige Regulatoren der Genexpression sind.

\section{ncRNA und „transcriptomics“}

Das menschliche Transkriptom, also die Gesamtheit aller RNA-Moleküle, die vom humanen Genom abgeschrieben werden, konnte erst in den letzten Jahren durch zwei neue Techniken beschrieben und charakterisiert werden:

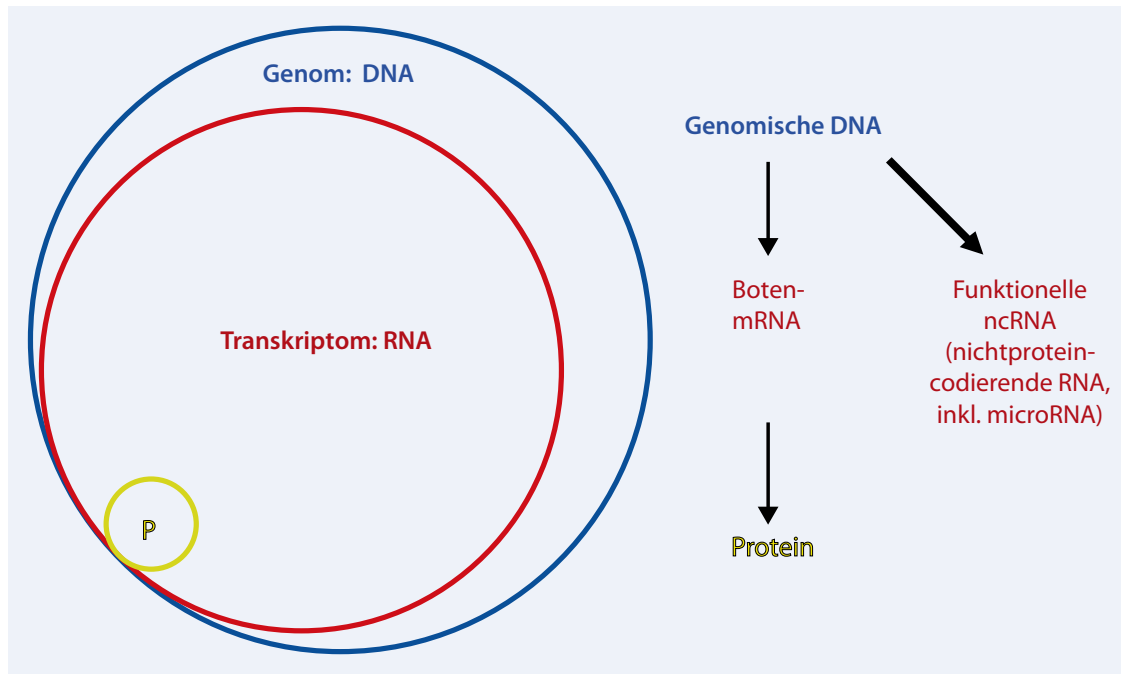

Abb. $1 \Delta$ Informationsgehalt des humanen Genoms. Das menschliche Genom (blau) wird zu etwa $70 \%$ in RNA transkribiert (rot), wohingegen nur etwa $2 \%$ der Genomsequenz für die Synthese von Proteinen kodieren $(\mathrm{gel} / \mathrm{b})$. Ein Großteil des Genoms wird daher in verschiedene Klassen nichtcodierender RNA (ncRNA) umgeschrieben, von denen die microRNAs eine der bekanntesten sind. Um die Information humaner Genomprojekte umfassend zu nutzen, bietet das Transkriptom daher eine deutlich gröBere Ressource für die Entdeckung neuer Biomarker als das Proteom
- „deep sequencing“,

- „tiling arrays“.

Beide Methoden erlauben es erstmals, große Mengen an Nukleotidsequenzen parallel zu analysieren. Beim so genannten „deep sequencing“ („tiefes Sequenzieren“, auch bekannt als „next generation sequencing“, „ultradeep sequencing“ oder „massively parallel sequencing“) werden viele Hunderttausend Nukleotidsequenzen aus einer einzigen Probe gleichzeitig ausgelesen. Wird dafür genomische DNA verwendet, so kann das humane Genom eines Individuums in kurzer Zeit vollständig analysiert werden. Die Methode ist aber genauso mit cDNA, aus RNA revers transkribierter komplementärer (c)DNA, verwendbar. So können auch alle Transkripte, das Transkriptom der Zelle, gleichzeitig sequenziert werden $[14,32,34,35]$.

„Tiling arrays“ sind Weiterentwicklungen klassischer Mikroarrays, wie sie schon lange zur Erstellung von Genexpressionsprofilen verwendet werden. Statt spezifischer Sonden für bekannte Gene auf klassischen Mikroarrays, sind auf „tiling arrays" allerdings Sonden aller Bereiche des Genoms in einem festen Abstand hinterlegt, so dass auch Transkripte aus Bereichen des Genoms detektiert werden können, in denen vorher keine Transkripte bekannt waren $[1,7,8,29]$.

Beide Methoden zur Entschlüsselung des humanen Transkriptoms führten unabhängig voneinander zum glei- 
chen Ergebnis: Ein weitaus größerer Teil des menschlichen Genoms wird in RNA umgeschrieben als vorher angenommen, wobei ncRNAs den weitaus größten Teil ausmachen. Schätzungen gehen davon aus, dass bis zu 70\% der Sequenz des humanen Genoms transkribiert werden, aber nur maximal $2 \%$ des menschlichen Genoms dienen als Baupläne für Proteine ( $\bullet$ Abb. 1). Der weitaus größte Teil des Genoms wird also nicht zur Herstellung proteincodierender mRNA, sondern für ncRNA abgelesen $[1,7,8,14,29$, $32,34,35]$.

\section{ncRNA: Transkriptionelles Rauschen oder funktionell wichtige Moleküle?}

Die bloße Existenz dieser vielen verschiedenen ncRNAs beweist selbstverständlich noch nicht deren funktionelle Bedeutung oder ob sie einen bedeutsamen Informationsgehalt besitzen. Es mehren sich aber die Hinweise darauf, dass sich unter den ncRNAs viele Moleküle mit wichtigen physiologischen oder pathologischen Funktionen befinden. Darüber hinaus bleibt festzuhalten, dass die molekulare Funktion nicht notwendigerweise den Informationsgehalt eines Markers vorhersagt: Ein diagnostischer oder prognostischer Marker wäre auch dann von größtem Interesse und praktischer Relevanz, wenn er nur mit einer bestimmten Erkrankung, Prognose oder Therapieansprechen signifikant korreliert, auch wenn er dort keine funktionelle Rolle spielen würde.

Anzeichen und Argumente für eine funktionelle Bedeutung vieler ncRNAs können wie folgt beschrieben werden:

Als erstes sind hier die microRNAs zu nennen, eine Klasse kleiner ncRNAs, die unzweifelhaft eine wichtige Funktion in der Regulation der Expression großer mRNAs spielen und in vielen Krankheiten dereguliert sind (s. unten). Aber auch großen ncRNAs wurden schon wichtige Funktionen zugeordnet: Die ncRNA XIST ist essenziell für die Stilllegung des zweiten X-Chromosoms in weiblichen Zellen $[3,4]$. Die ncRNA HOTAIR beeinflusst andere Gene über die Regulation der Chromatinstruktur [16, 22, 30].

Zusätzlich deutet der dreifache Aufwand, den die Zelle in die ncRNAs inves-

Pathologe 2010 · [Suppl 2] 31:258-262 DOI 10.1007/s00292-010-1336-8

c) Springer-Verlag 2010

\section{S. Diederichs \\ Nichtcodierende RNA in malignen Tumoren. Eine neue Welt von Tumor-Biomarkern und Zielstrukturen in Krebszellen}

\section{Zusammenfassung}

Das menschliche Genom enthält nur zu etwa $2 \%$ proteincodierende Gene, auf die sich die medizinische Forschung der letzten Jahrzehnte maßgeblich konzentriert hat. Da aber bis zu 70\% des humanen Genoms in RNA transkribiert werden, enthält das Genom weit mehr nichtcodierende Information, die als nichtcodierende RNA (ncRNA) in der Zelle vorliegen. Viele dieser ncRNAs sind stark exprimiert, spezifisch reguliert und evolutionär konserviert, was für ihre funktionelle Bedeutung spricht. Die microRNAs sind die bekanntesten ncRNAs, daneben existieren jedoch viele andere, lange ncRNAs. Differenzielle ncRNA- oder microRNA-Expressionsmuster korrelieren in vielen Tumorentitäten mit der Diagnose oder Prognose und kön- nen daher als umfangreiche Quelle für neue Biomarker dienen. Die Expression der langen ncRNA MALAT1 ist in Lungen-, Brust- und Leberkrebs assoziiert mit der Entstehung des Tumors, der Tumorprogression oder dem Überleben. Funktionell aktive ncRNAs erlauben neue Einblicke in die Entstehungsmechanismen von Tumoren. Die Summe der verschiedenen, oft noch unbekannten ncRNAs lässt wichtige Entdeckungen und neue Impulse in Diagnostik, Prognostik und Therapie vieler Erkrankungen erwarten.

Schlüsselwörter

Transkriptom · Biomarker · Nichtcodierende RNA $\cdot$ microRNA $\cdot$ MALAT1

\section{Non-coding RNA in malignant tumors. A new world of tumor biomarkers and target structures in cancer cells}

\section{Abstract}

Only about $2 \%$ of the human genome constitute protein-coding genes - nevertheless, medical research has focused mainly on this portion in recent decades. Since up to $70 \%$ of the human genome is transcribed into RNA, the genome contains much more non-coding information than coding, which is present in the cell as non-coding RNA (ncRNA). Many of these ncRNAs are highly expressed, specifically regulated and evolutionarily conserved, arguing in favor of their functional significance. MicroRNAs are the most well-known ncRNAs, but many other long ncRNAs exist. Differential ncRNA or microRNA expression patterns correlate with diagnosis or progno- sis in many tumor entities and can thus serve as an extensive source of new biomarkers. The expression of the long ncRNA MALAT1 correlates with tumor development, progression or survival in lung, liver and breast cancer. Functionally active ncRNAs can provide novel insights into the mechanisms underlying tumor development. The large number of different, often as yet unidentified ncRNAs promises new stimuli for the diagnosis, prognosis and therapy of many diseases.

\section{Keywords}

Transcriptomes · Biomarkers · Non-coding RNA $\cdot$ microRNA MALAT1 
tiert, darauf hin, dass diese Moleküle von großer Bedeutung für die Zelle sind:

- Es kostet die Zelle viel Energie, die ncRNAs herzustellen.

- In vielen Geweben findet man spezifische Expressionsmuster von ncRNAs [28], die auf eine aktive Regulation der ncRNAs und damit wiederum einen Aufwand für die Zelle hindeuten.

- Einige ncRNAs sind auch außerordentlich gut evolutionär konserviert (z. B. MALAT1, s. unten), so dass eine Mutation in diesen ncRNA-Genen für die Zelle nachteilig erscheint.

Daher erscheint insgesamt die Hypothese gerechtfertigt, dass viele ncRNAs in der Zelle unter physiologischen oder pathologischen Bedingungen wichtige Funktionen erfüllen können.

\section{Das Komplexitätsparadoxon}

Ein weiteres interessantes Phänomen in Verbindung mit dem Anteil nichtcodierender DNA am Genom ist das so genannte „Komplexitätsparadoxon“. Das Paradoxon besteht darin, dass sich einerseits verschiedene Spezies von der Hefe (Saccharomyces cerevisiae) über den Fadenwurm (Caenorhabditis elegans) bis zum Menschen in ihrer Komplexität, z. B. in der Anzahl an Zellen oder verschiedenen Gewebe pro Organismus, deutlich unterscheiden. Andererseits ist die Anzahl der Gene zwischen einfacheren und komplexeren Organismen nicht im gleichen Maße unterschiedlich. Zusätzlich haben viele proteincodierende Gene der komplexen Organismen Orthologe in den weniger komplexen Spezies.

Woher kommt also die zusätzliche Information, die die Komplexität eines Säugetierorganismus von der Hefe unterscheidet? Die Antwort darauf überrascht: Es gibt über alle Spezies hinweg eine klare Korrelation zwischen dem Gehalt des Genoms an nichtcodierenden Sequenzen und der Komplexität des Organismus [26]. Auch dieses Phänomen deutet auf die Signifikanz des nichtcodierenden Anteils des Genoms hin.

\section{microRNA}

Eine spezielle Klasse von ncRNAs sind die microRNAs, die mit über 8000 publizierten Arbeiten bereits die Aufmerksamkeit der breiten wissenschaftlichen Öffentlichkeit erlangt haben. Eine microRNA ist eine sehr kurze ncRNA von nur etwa 21 Nukleotiden, die die Stabilität oder Translation von vielfach größeren proteincodierenden mRNAs hemmen kann [37]. MicroRNAs stellen somit wichtige Regulatoren der Genexpression dar. Viele microRNAs wurden bereits mit der Entstehung von Krebserkrankungen in Verbindung gebracht, so z. B. die microRNA let-7, die das bekannte Onkogen Ras reguliert [21].

Fehlregulation und spezifische Expressionsmuster von microRNAs, die auch diagnostische und prognostische Anwendungen finden können, wurden in vielen verschiedenen Tumorentitäten beschrieben [6, 24], funktionell bedeutsame Mutationen von microRNAs sind hingegen deutlich seltener in Tumoren $[6,10]$. MicroRNA-Expressionsmuster können auch als Biomarker verwendet werden [18, 38] und sind ebenso aus Plasma oder Serum detektierbar [18].

Die reife microRNA ist das Produkt eines komplexen Prozessierungsweges, in dem aus einem langen Vorläufermolekül eine kleine, aktive microRNA entsteht [37]. Die microRNA-Maschinerie der Zelle stellt aber auch die Grundlage für das gezielte Abschalten spezifischer Gene mit kleinen künstlichen RNAs dar, so genannte siRNAs, mit der Methode der RNA-Interferenz (RNAi; [13]). Daher ist ein genaues Verständnis der Rolle von microRNAs nicht nur diagnostisch bedeutsam, sondern kann auch zur Verbesserung von Therapieansätzen mittels RNAi dienen $[9,11]$.

\section{Lange ncRNA als Tumormarker}

Neben microRNAs zeigen auch verschiedene lange ncRNAs (abgekürzt mit lincR$\mathrm{NA}$, „long intergenic non-coding RNA“) signifikante Expressionsunterschiede zwischen gesundem und malignem Gewebe des gleichen Organs.

Als ein Beispiel wird hier MALATı näher beschrieben. Die lincRNA MALAT1 wurde erstmals 2003 beschrieben und in einer subtraktiven Hybridisierung als Markergen beim Lungenkrebs entdeckt. Die Expression von MALAT1 in frühen Stadien des Adenokarzinoms korrelierte signifikant mit dem Überleben der Patienten und war prädiktiv für die spätere Entwicklung von Fernmetastasen nach Resektion des Primärtumors [20].

Nach der erstmaligen Beschreibung wurde MALAT 1 in vielen Tumorentitäten differenziell exprimiert gefunden, so in Brust- oder Lebertumoren [23, 25, 33]. Es ist daher ein interessanter Kandidat für weitergehende Studien zu seiner diagnostischen oder prognostischen Bedeutung [15].

MALAT1 ist 8000 Nukleotide lang und evolutionär außergewöhnlich gut konserviert [20]. Das 3'-Ende von MALAT1 wird durch einen besonderen Mechanismus generiert [36].

Die molekulare Funktion von MALAT1 ist aber noch nach wie vor ungeklärt.

Frühe Beispiele für tumorassoziierte ncRNAs waren auch die lincRNAs H19 [2, 17] und BIC [12, 31], die beide aber auch zu kurzen microRNAs weiter prozessiert werden können, H19 zu miR-675 [5] und BIC zu miR-155 [12, 31]. Ob die Funktion dieser lincRNAs primär in der Herstellung der enthaltenen microRNAs liegt oder ob die langen Vorläufer selbst noch funktionell aktiv sind, ist aber noch ungeklärt.

\section{Vor- und Nachteile von RNA-Biomarkern}

Zunächst erscheinen RNA-Moleküle aufgrund ihrer Instabilität nicht als ideale Biomarker, da sie unter großer Sorgfalt und am besten aus Frisch- oder Gefriergewebe isoliert werden sollten, um den RNAAbbau durch RNasen, RNA-abbauende Enzyme, zu verhindern. Durch neue Extraktionstechniken, Automatisierung und die Verwendung von RNA-schützenden Agenzien ist diese Problematik aber beherrschbar.

Die Klasse der microRNAs ist in diesem Punkt wiederum von besonderem Interesse, da diese kleinen RNAs im Vergleich zu großen RNA-Molekülen eine deutlich höhere Stabilität aufweisen. Die RNA kann sowohl in morphologischen Schnitten durch In-situ-Hybridisierung 
(ISH) als auch durch quantitative „real-time" Polymerase-Ketten-Reaktion (qPCR) nach reverser Transkription (RT) der RNA in cDNA nachgewiesen werden. Die qPCR offenbart zwei große Vorteile:

- Die Methode ist quantitativ und erlaubt anhand von Standardverdünnungsreihen die objektive Quantifikation der in einer Probe vorhandenen Menge einer bestimmten RNA.

- RNA bzw. die revers transkribierte cDNA ist mittels PCR-Techniken amplifizierbar, um auch sehr geringe RNA-Mengen gut detektieren zu können, so dass die Sensitivität von RNA im Vergleich zu Protein-Biomarkern höher ist und das Spektrum beobachtbarer Gene erheblich erweitert wird.

Funktionelle ncRNAs eignen sich wiederum besonders gut als RNA-Biomarker, da die Messung ihrer Expression direkte Aussagen über die Abundanz des aktiven Moleküls erlaubt. Bei der Analyse proteincodierender mRNA wird hingegen ledig- lich eine Vorstufe des funktionell aktiven Proteins detektiert, dessen Aktivität dann durch Translationseffizienz, posttranslationale Modifikationen und Proteinabbau beeinflusst wird.

\section{ncRNA als funktionelle Moleküle in Krebszellen}

Neben ihrer möglichen Bedeutung als Quelle für neue Biomarker können ncRNAs aber auch wichtige Funktionen in der Zelle übernehmen.

Während die Funktion von MALAT1 noch weitgehend unbekannt ist, wurden kürzlich wichtige zelluläre Funktionen für andere lange ncRNA beschrieben [27]. Hier soll nur ein Beispiel beschrieben werden: „HOTAIR-“ oder „HOX-antisense-intergenic-RNA“. HOTAIR ist eine lange intergenische ncRNA (lincRNA), die die Chromatinstruktur des HOX-Genclusters regulieren kann [30]. Die Expression von HOTAIR kann über die Regulation der Genexpression auch die Metastasierung maligner Tumoren beschleu- nigen [16]. HOTAIR interagiert dazu mit dem Protein PRC2, wie es auch viele andere lincRNAs tun, die entsprechend auch Chromatinstrukturen und die Genexpression regulieren könnten $[22,30]$. Dies zeigt, dass ncRNAs wichtige Funktionen in der Zelle ausführen können und für die Entstehung und den Verlauf maligner Erkrankungen verantwortlich sein können.

Fazit

Daten über das menschliche Genom und das menschliche Transkriptom werden in den nächsten Jahren in ungeahntem Ausmaß zur Verfügung stehen. Die Genome und Transkriptome vieler Tumoren werden derzeit schon im „International Cancer Genome Consortium“ sequenziert [19]. Es ist nicht unrealistisch, dass Genom- und Transkriptomdaten noch in diesem Jahrzehnt für jeden Tumorpatienten zur Verfügung stehen. Um diese Informationsmenge vollständig ausnutzen zu können, ist es unerlässlich, nicht nur den kleinen Teil der proteincodieren-

\section{Hier steht eine Anzeige.}

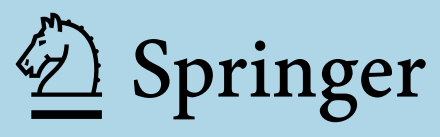


den Gene zu berücksichtigen, sondern auch den weit größeren Teil des Genoms, der nichtcodierende Information enthält. Erste Beispiele für wichtige ncRNAs mit diagnostischer, prognostischer und funktioneller Bedeutung stehen mit den microRNAs, MALAT1 und HOTAIR zur Verfügung. Gemessen an der Vielzahl der noch nicht untersuchten ncRNAs sind noch viele große Entdeckungen zu erwarten. Nun gilt es, diese reiche Quelle der Information auch für die Pathologie zu nutzen.

\section{Korrespondenzadresse \\ Dr. S. Diederichs}

Helmholtz-Hochschul-Gruppe „Molekulare RNA Biologie \& Krebs", Deutsches Krebsforschungszentrum (DKFZ) \& Pathologisches Institut Universität Heidelberg

Im Neuenheimer Feld 280 (B150),

69120 Heidelberg

s.diederichs@dkfz.de

Interessenkonflikt. Der korrespondierende Autor gibt an, dass kein Interessenkonflikt besteht.

\section{Literatur}

1. Bertone P, Stolc V, Royce TE et al (2004) Global identification of human transcribed sequences with genome tiling arrays. Science 306:2242-2246

2. Brannan Cl, Dees EC, Ingram RS et al (1990) The product of the $\mathrm{H} 19$ gene may function as an RNA. Mol Cell Biol 10:28-36

3. Brockdorff N, Ashworth A, Kay GF et al (1992) The product of the mouse Xist gene is a $15 \mathrm{~kb}$ inactive $X$-specific transcript containing no conserved ORF and located in the nucleus. Cell 71:515-526

4. Brown CJ, Hendrich BD, Rupert JL et al (1992) The human XIST gene: analysis of a $17 \mathrm{~kb}$ inactive $X-$ specific RNA that contains conserved repeats and is highly localized within the nucleus. Cell 71:527542

5. Cai X, Cullen BR (2007) The imprinted H19 noncoding RNA is a primary microRNA precursor. RNA 13:313-316

6. Calin GA, Ferracin M, Cimmino A et al (2005) A MicroRNA signature associated with prognosis and progression in chronic lymphocytic leukemia. N Engl J Med 353:1793-1801

7. Carninci P, Kasukawa T, Katayama S et al (2005) The transcriptional landscape of the mammalian genome. Science 309:1559-1563

8. Cheng J, Kapranov P, Drenkow J et al (2005) Transcriptional maps of 10 human chromosomes at 5nucleotide resolution. Science 308:1149-1154

9. Diederichs S, Haber DA (2007) Dual role for argonautes in microRNA processing and posttranscriptional regulation of microRNA expression. Cell 131:1097-1108

10. Diederichs S, Haber DA (2006) Sequence variations of microRNAs in human cancer: alterations in predicted secondary structure do not affect processing. Cancer Res 66:6097-6104
11. Diederichs $S$, Jung $S$, Rothenberg SM et al (2008) Coexpression of argonaute-2 enhances RNA interference toward perfect match binding sites. Proc Natl Acad Sci U S A 105:9284-9289

12. Eis PS, Tam W, Sun L et al (2005) Accumulation of miR-155 and BIC RNA in human B cell lymphomas. Proc Natl Acad Sci U S A 102:3627-3632

13. Elbashir SM, Harborth J, Lendeckel W et al (2001) Duplexes of 21-nucleotide RNAs mediate RNA interference in cultured mammalian cells. Nature 411:494-498

14. ENCODE (2009) Post-transcriptional processing generates a diversity of $5^{\prime}$-modified long and short RNAs. Nature 457:1028-1032

15. Fellenberg J, Bernd L, Delling G et al (2007) Prognostic significance of drug-regulated genes in high-grade osteosarcoma. Mod Pathol 20:10851094

16. Gupta RA, Shah N, Wang KC et al (2010) Long noncoding RNA HOTAIR reprograms chromatin state to promote cancer metastasis. Nature 464:10711076

17. Hao Y, Crenshaw T, Moulton T et al (1993) Tumoursuppressor activity of H19 RNA. Nature 365:764767

18. Hu Z, Chen X, Zhao Y et al (2010) Serum microRNA signatures identified in a genome-wide serum microRNA expression profiling predict survival of non-small-cell lung cancer. J Clin Oncol 28:17211726

19. ICGC (2010) International network of cancer genome projects. Nature 464:993-998

20. Ji P, Diederichs S, Wang W et al (2003) MALAT-1, a novel noncoding RNA, and thymosin beta4 predict metastasis and survival in early-stage non-small cell lung cancer. Oncogene 22:8031-8041

21. Johnson SM, Grosshans H, Shingara J et al (2005) RAS is regulated by the let-7 microRNA family. Cell 120:635-647

22. Khalil AM, Guttman M, Huarte M et al (2009) Many human large intergenic noncoding RNAs associate with chromatin-modifying complexes and affect gene expression. Proc Natl Acad Sci U S A 106:11667-11672

23. Lin R, Maeda S, Liu C et al (2007) A large noncoding RNA is a marker for murine hepatocellular carcinomas and a spectrum of human carcinomas. Oncogene 26:851-858

24. Lu J, Getz G, Miska EA et al (2005) MicroRNA expression profiles classify human cancers. Nature 435:834-838

25. Luo JH, Ren B, Keryanov S et al (2006) Transcriptomic and genomic analysis of human hepatocellular carcinomas and hepatoblastomas. Hepatology 44:1012-1024

26. Mattick JS (2004) RNA regulation: a new genetics? Nat Rev Genet 5:316-323

27. Mercer TR, Dinger ME, Mattick JS (2009) Long noncoding RNAs: insights into functions. Nat Rev Genet 10:155-159

28. Mercer TR, Dinger ME, Sunkin SM et al (2008) Specific expression of long noncoding RNAs in the mouse brain. Proc Natl Acad Sci U S A 105:716-721

29. Rinn JL, Euskirchen G, Bertone P et al (2003) The transcriptional activity of human Chromosome 22. Genes Dev 17:529-540

30. Rinn JL, Kertesz M, Wang JK et al (2007) Functional demarcation of active and silent chromatin domains in human HOX loci by noncoding RNAs. Cell 129:1311-1323

31. Rodriguez A, Vigorito E, Clare S et al (2007) Requirement of bic/microRNA-155 for normal immune function. Science 316:608-611
32. Sultan M, Schulz MH, Richard H et al (2008) A global view of gene activity and alternative splicing by deep sequencing of the human transcriptome. Science 321:956-960

33. Sun Y, Wu J, Wu SH et al (2009) Expression profile of microRNAs in c-Myc induced mouse mammary tumors. Breast Cancer Res Treat 118:185-196

34. Suzuki H, Forrest AR, Van Nimwegen E et al (2009) The transcriptional network that controls growth arrest and differentiation in a human myeloid leukemia cell line. Nat Genet 41:553-562

35. Wang ET, Sandberg R, Luo S et al (2008) Alternative isoform regulation in human tissue transcriptomes. Nature 456:470-476

36. Wilusz JE, Freier SM, Spector DL (2008) 3' end processing of a long nuclear-retained noncoding RNA yields a tRNA-like cytoplasmic RNA. Cell 135:919932

37. Winter J, Jung S, Keller S et al (2009) Many roads to maturity: microRNA biogenesis pathways and their regulation. Nat Cell Biol 11:228-234

38. Yang N, Kaur S, Volinia S et al (2008) MicroRNA microarray identifies Let-7i as a novel biomarker and therapeutic target in human epithelial ovarian cancer. Cancer Res 68:10307-10314 